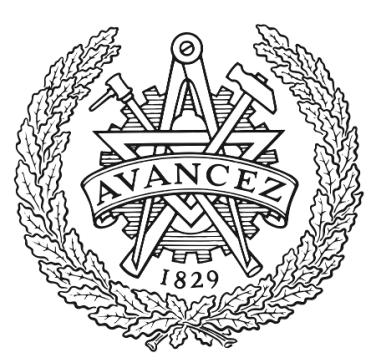

CHALMERS

UNIVERSITY OF TECHNOLOGY

\title{
Implementation of a Quasi-Three-Dimensional Nonreflecting Blade Row Interface for Steady and Unsteady Analysis of Axial Turbomachines
}

Downloaded from: https://research.chalmers.se, 2023-04-26 00:05 UTC

Citation for the original published paper (version of record):

Lindblad, D., Montero Villar, G., Andersson, N. et al (2018). Implementation of a

Quasi-Three-Dimensional Nonreflecting Blade Row Interface for Steady and Unsteady Analysis of Axial Turbomachines. Turbomachinery and Core Noise, 5. http://dx.doi.org/10.2514/6.2018-4187

N.B. When citing this work, cite the original published paper. 


\title{
Implementation of a Quasi-Three-Dimensional Nonreflecting Blade Row Interface for Steady and Unsteady Analysis of Axial Turbomachines
}

\author{
Daniel Lindblad*, Gonzalo Montero Villar ${ }^{\dagger}$, and Niklas Andersson * \\ Chalmers University of Technology, Gothenburg, SE-412 96, Sweden \\ Nathan A. Wukie $\S$ \\ University of Cincinnati, Cincinnati, Ohio, 45221
}

\begin{abstract}
Higher order nonreflecting blade row interfaces are today widely used for performing both steady and unsteady simulations of the flow withing axial turbomachines. In this paper, a quasithree-dimensional nonreflecting interface based on the exact, two-dimensional nonreflecting boundary condition for a single frequency and azimuthal wave number developed by Giles is presented. The formulation has been implemented to work for both steady simulations as well as unsteady simulations employing the nonlinear Harmonic Balance method. The theory behind the construction of the nonreflecting interface is presented and details on the numerical implementation is also provided. The implementation is verified for two dimensional wave propagation along a straight cascade. It is shown that the interface correctly absorbs incoming waves, but also found that the chosen implementation strategy may be ill-posed. A simple solution to stabilize the implementation is therefore implemented, but future work should seek a more generic solution to this problem.
\end{abstract}

Roman/Greek

\section{Nomenclature}

$\bar{A} \quad=$ axial gradient flux Jacobian

$c \quad=$ speed of sound, $\mathrm{m} \mathrm{s}^{-1}$

$\bar{C} \quad=$ circumferential gradient flux Jacobian

$i \quad=$ imaginary unit

$k_{x}=$ axial wave number and eigenvalue, $\mathrm{rad} \mathrm{m}^{-1}$

$k_{z} \quad=$ azimuthal wave number, $\mathrm{rad} \mathrm{m}^{-1}$

$m_{n, k} \quad=$ nodal diameter

$n \quad=$ direction of face normal

$N=$ number of blades or number of points per wavelength

$N_{a} \quad=$ number of azimuthal harmonics

$N_{h} \quad=$ number of temporal harmonics

$N_{t} \quad=$ number of time levels

$p \quad=$ pressure, $\mathrm{kg} \mathrm{m}^{-1} \mathrm{~s}^{-2}$

$P \quad=$ pitch of blade row, $\mathrm{m}$

$q=$ vector containing primitive variables

$\hat{q} \quad=$ eigenvector

$\hat{q}_{n} \quad=\quad$ vector containing temporal Fourier transform of primitive variables

$\hat{q}_{n, k}=$ vector containing temporal-circumferential Fourier transform of primitive variables

$t \quad=$ time, $\mathrm{s}$

$T=$ matrix containing eigenvectors sorted into its columns

\footnotetext{
*Ph.D. Student, Department of Mechanics and Maritime Sciences, Hörsalsvägen 7A, SE-412 96 Gothenburg.

${ }^{\dagger}$ Ph.D. Student, Department of Mechanics and Maritime Sciences, Hörsalsvägen 7A, SE-412 96 Gothenburg.

$¥$ Assistant Professor, Department of Mechanics and Maritime Sciences, Hörsalsvägen 7A, SE-412 96 Gothenburg.

${ }^{\S}$ Ph.D. Student, Department of Aerospace Engineering, ML 70, Cincinnati, Ohio 45221, AIAA Student Member.
} 


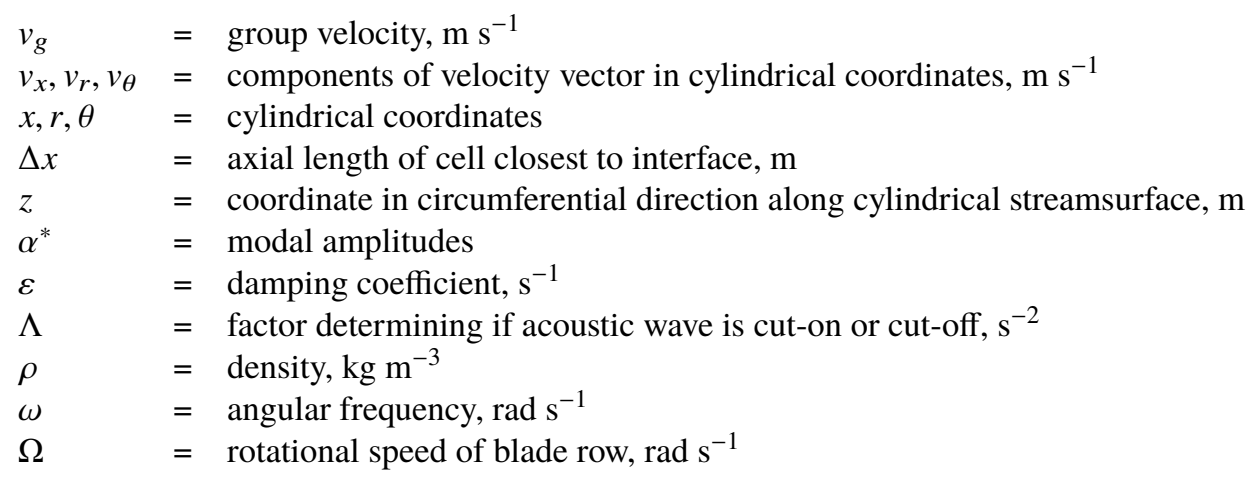

\section{Subscripts/Superscripts}

$\begin{array}{ll}\square_{\text {int }} & =\text { interior state } \\ \square_{\text {ext }} & =\text { exterior state } \\ \square^{\prime} & =\text { perturbation } \\ \square & =\text { temporal+circumferential average }\end{array}$

\section{Introduction}

TheE dimensional simulations of axial turbomachinery flows based on numerical solutions of the nonlinear Reynolds Averaged Navier Stokes equations are today commonplace within both academia and industry. Often, these simulations consist of several blade rows in relative motion coupled by so called blade row interfaces. The most common interface in use today is the mixing plane for which radial profiles of circumferentially averaged flow properties are transferred between the blade rows. This approach allows steady simulations to be performed efficiently, since azimuthal variations in the flow are mixed out before being transfered across the interface. Time accurate simulations employing a sliding grid interface to couple the blade rows does on the other hand enable unsteady blade row interactions to be included in the analysis, but are also much more computationally demanding [1]. In recent years, the nonlinear Harmonic Balance method [2] and the nonlinear Frequency Domain method [3] have therefore gained significant attention as efficient alternatives for performing unsteady simulations of turbomachinery blade row interactions. When these methods are used, a mode matching condition which only transfers resolved frequencies between the blade rows is commonly employed [1, 4, 5].

For simulations employing either a mixing plane or a mode matching condition the solution often appears discontinuous across the interface. This occurs because only a limited amount of all the information available on one side of the interface is transferred to the other side when these methodologies are employed [1]. This discontinuity must be properly addressed in the formulation of the interface if spurious reflections are to be prevented from being generated at the interface. In this work, the theory behind the exact, two dimensional nonreflecting boundary condition for a single frequency and azimuthal wave number developed by Giles [6, 7], whose work in turn is based on the early work of Engquist and Majda [8], has been adopted for this purpose. In particular, the aim of the current work has been to implement a nonreflecting interface formulation, which is applicable to both steady simulations as well as unsteady simulations using the Harmonic Balance method. When the formulation is applied to unsteady simulations it is nonlocal in both space and time. This is however not a problem in the context of the Harmonic Balance method since the entire time period then is stored in the solution. It should also be noted that we employ the method proposed by Saxer and Giles [9] for extending the two dimensional theory developed by Giles to three dimensional flows.

The rest of this paper is structured as follows. First, an overview of how a mode matching interface that does not employ any nonreflecting treatment may be implemented is presented. This is used to highlight the necessity of adding nonreflecting treatment to the interface in order to avoid spurious reflections. After this, the theory necessary for constructing the nonreflecting interface developed in this work is covered in detail. An algorithm for implementing this interface is also provided and its implications on the stability of the numerical scheme are briefly discussed. Lastly, the implementation of the nonreflecting interface into the Chalmers developed G3D::Flow code is verified for two dimensional wave propagation inside a straight cascade. 


\section{Method}

\section{A. Mode Matching Condition at Blade Row Interface}

Turbomachinery blade rows in relative motion generate an unsteady flow field that can be represented by a set of circumferential lobes, each one having a certain nodal diameter and corresponding frequency with which it rotates. In this work we only consider turbomachines that consist of two blade rows, for which case the circumferential lobes will attain the following nodal diameters [4, 10]

$$
m_{n, k}=k N_{1}+n N_{2}
$$

Here, $N_{1}$ and $N_{2}$ represent the number of blades in the first and second blade row respectively. In G3D::Flow, the flow in each blade row is simulated in the relative frame of reference. This causes a lobe defined by Eq. (1) to rotate at a different frequency in each relative frame equal to the relative blade passing frequency of the adjacent blade row [5]

$$
\begin{aligned}
& \omega_{n, 1}=n N_{2}\left(\Omega_{2}-\Omega_{1}\right) \\
& \omega_{k, 2}=k N_{1}\left(\Omega_{1}-\Omega_{2}\right)
\end{aligned}
$$

In this equation, $\Omega_{1}$ and $\Omega_{2}$ denote the rotational speed of the first and second blade row respectively. The periodic flow defined by Eqs. (1) and (2) may be represented by a double Fourier series expansion as follows [5]

$$
\begin{aligned}
& q(x, r, \theta, t)=\sum_{n=-N_{h}}^{N_{h}} \sum_{k=-N_{a}}^{N_{a}} \hat{q}_{n, k}(x, r) e^{i\left(\omega_{n, 1} t-m_{n, k} \theta\right)} \\
& q(x, r, \theta, t)=\sum_{n=-N_{a}}^{N_{a}} \sum_{k=-N_{h}}^{N_{h}} \hat{q}_{n, k}(x, r) e^{i\left(\omega_{k, 2} t-m_{n, k} \theta\right)}
\end{aligned}
$$

Here, $N_{h}$ and $N_{a}$ represent the number of temporal and azimuthal harmonics that are included in the series expansion and $q=\left(\rho, v_{x}, v_{r}, v_{\theta}, p\right)^{T}$ is a vector that contains the primitive flow variables in cylindrical coordinates and the absolute frame of reference. The time-azimuthal Fourier coefficients in Eq. (3) are obtained by integrating the temporal Fourier coefficients of the primitive flow variables according to

$$
\begin{aligned}
& \hat{q}_{n, k}(x, r)=\frac{N_{1}}{2 \pi} \int_{0}^{2 \pi / N_{1}} \hat{q}_{n}(x, r, \theta) e^{i m_{n, k} \theta} d \theta \\
& \hat{q}_{n, k}(x, r)=\frac{N_{2}}{2 \pi} \int_{0}^{2 \pi / N_{2}} \hat{q}_{k}(x, r, \theta) e^{i m_{n, k} \theta} d \theta
\end{aligned}
$$

Furthermore, the temporal Fourier coefficients can be calculated in either of two ways depending on if the flow is simulated using the time-accurate solver [5] or the time-spectral Harmonic Balance method [2] available in G3D::Flow. In the first case, a moving average technique [11] is used to update the coefficients, and in the latter case they are obtained from a discrete Fourier transform over all $N_{t}=2 N_{h}+1$ time levels that constitute the Harmonic Balance solution.

The purpose of the blade row interface used in this work is to ensure that the time-azimuthal Fourier coefficients obtained on each side of the interface match. In the original implementation of the interface described in [12] this was accomplished according to the method presented by Olausson [5], which in turn is similar to the interface treatment developed by Gerolymos et al. [11] for the Chorochronic method and Ekici et al. [4, 13] for the Harmonic Balance method. In this implementation it is assumed that the mesh on each side of the interface is divided into bands of faces with constant radius. For each of these bands the flow field is first sampled according to Eq. (4) to obtain a set of time-azimuthal Fourier coefficients on both sides of the interface. Coefficients obtained at a certain radial location on one side of the interface are then used to calculate an exterior state for all cells at the same radial location on the other side of the interface using Eq. (3). Once this exterior state has been obtained, the flux over these cells may finally be calculated. This way, the flow state on each side of the interface is driven to match that sampled on the other side.

Matching of Fourier coefficients, which we hereinafter will refer to as mode matching, can however only be performed for those coefficients that are resolved on both sides of the interface, i.e. for Fourier coefficients whose indices satisfy $n, k \leq \min \left(N_{h}, N_{a}\right)$ c.f. Eq. (3). This causes the solution to be discontinuous across the interface with respect to all flow perturbations generated by the higher harmonics, which in turn may yield spurious reflections. A simple solution to this problem would be to increase the number of temporal and azimuthal harmonics sampled on 
both sides of the interface until all relevant flow perturbations are included in the sampling. This may however not always be possible, especially with the Harmonic Balance method for which the computational cost increases with the number of time levels $\left(2 N_{h}+1\right)$. In these cases different strategies have therefore been developed to ensure that the discontinuous flow field does not cause any spurious reflections. One approach is to treat all Fourier coefficients which do not have a counter part in the adjacent blade row using the nonreflecting boundary condition theory of Giles [6, 7] for two dimensional flows and the extension of Giles' theory to three dimensional flows proposed by Saxer and Giles [9]. This approach has been applied to two dimensional flows by e.g. Ekici et al. [4, 13]. In addition to this it is also possible to formulate the mode matching condition at the interface in terms of the nonreflecting boundary condition theory as was done for three dimensional flows by e.g. Frey et al. [1]. In this work we follow the latter strategy and formulate a mode matching interface which treats all Fourier coefficients in a consistent way based on the nonreflecting theory of Giles [6, 7] and Saxer and Giles [9]. In the upcoming sections the nonreflecting theory developed by these authors will be reviewed and we will then proceed to define our mode matching algorithm.

\section{B. Linearized Euler Equations in Two Dimensions}

A fundamental assumption behind the nonreflecting theory developed by Giles [6, 7] is that the flow close to a boundary or interface obeys the linearized Euler equations in two dimensions. When this theory is extended to three dimensions one instead assumes that the flow along cylindrical surfaces with constant radius obeys the following form of the linearized Euler equations [9]

$$
\frac{\partial q^{\prime}}{\partial t}+\bar{A} \frac{\partial q^{\prime}}{\partial x}+\frac{1}{r} \bar{C} \frac{\partial q^{\prime}}{\partial \theta}=0
$$

Here, $q^{\prime}=q-\bar{q}$ denotes a perturbation of the primitive flow variables relative to a circumferentially+temporally averaged mean state $\bar{q}$. Note that the mean state corresponds to the zeroth Fourier coefficient in Eq. (4), which in turn implies that $q^{\prime}$ may be obtained by omitting $n, k=0$ in Eq. (3). The flux Jacobians $\bar{A}$ and $\bar{C}$ in Eq. (5) are further defined as follows

$$
\bar{A}=\left[\begin{array}{ccccc}
\bar{v}_{x} & \bar{\rho} & 0 & 0 & 0 \\
0 & \bar{v}_{x} & 0 & 0 & 1 / \bar{\rho} \\
0 & 0 & \bar{v}_{x} & 0 & 0 \\
0 & 0 & 0 & \bar{v}_{x} & 0 \\
0 & \gamma \bar{p} & 0 & 0 & \bar{v}_{x}
\end{array}\right] \quad \bar{C}=\left[\begin{array}{ccccc}
\bar{v}_{\theta} & 0 & 0 & \bar{\rho} & 0 \\
0 & \bar{v}_{\theta} & 0 & 0 & 0 \\
0 & 0 & \bar{v}_{\theta} & 0 & 0 \\
0 & 0 & 0 & \bar{v}_{\theta} & 1 / \bar{\rho} \\
0 & 0 & 0 & \gamma \bar{p} & \bar{v}_{\theta}
\end{array}\right]
$$

For a perfect ideal gas, $\gamma \bar{p}=\bar{\rho} \bar{c}^{2}$, where $\bar{c}$ is the average speed of sound and $\gamma$ the ratio of specific heats.

If Eq. (5) is to be a good representation of the flow it must hold that the perturbations are small in amplitude, viscous effects can be neglected, the mean flow varies slowly in both the axial and radial direction and that the mean velocity vector has a negligible radial component. It must also hold true that the flow perturbations do not vary in the radial direction, i.e. that $q^{\prime}$ consist of planar waves in the $(x, \theta)$-plane. The advantage of constructing the nonreflecting interface based on Eq. (5) is that the analysis can be performed independently at each radial position. If in contrast radial flow variations would be included in Eq. (5) the whole boundary must be considered simultaneously in the analysis [14]. This has the benefit of potentially improving the results, but also increases the complexity and computational cost of the implementation.

\section{Modal Decomposition based on Linearized Euler Equations}

The linear nature of Eq. (5) implies that each contribution to $q^{\prime}$ caused by a single time-azimuthal Fourier coefficient in Eq. (3) develops independently of the others, which in turn means that we can treat all the Fourier coefficients independently in the construction of the nonreflecting interface. A flow perturbation caused by a single Fourier coefficient is further assumed to be composed of waves on the following form

$$
q^{\prime}(x, r, \theta, t)=\hat{q}(r) e^{i\left(\omega t-k_{x} x-k_{z} z\right)}
$$

Here, $k_{x}$ denotes the axial wave number of the wave and $k_{z} z=m \theta$, where $k_{z}=m / r$ is the azimuthal wave number of the wave and $z=r \theta$. If the same convention is applied to Eq. [5], it will read as follows 


$$
\frac{\partial q^{\prime}}{\partial t}+\bar{A} \frac{\partial q^{\prime}}{\partial x}+\bar{C} \frac{\partial q^{\prime}}{\partial z}=0
$$

By combining Eqs. (7) and (8) one obtains

$$
\left(\omega-k_{x} \bar{A}-k_{z} \bar{C}\right) \hat{q}(r)=0
$$

From this equation an eigenvalue problem for $k_{x}$, with corresponding eigenvector $\hat{q}(r)$, may be derived by multiplying from the left by $\bar{A}^{-1}$

$$
\left(\omega \bar{A}^{-1}-k_{z} \bar{A}^{-1} \bar{C}-k_{x} I\right) \hat{q}(r)=0
$$

Note that this eigenvalue problem can only be constructed if $\bar{A}$ is invertible, which holds true if its determinant $\operatorname{det}(\bar{A})=\bar{v}_{x}^{3}\left(\bar{v}_{x}^{2}-\bar{c}^{2}\right)$ is nonzero. In order to satisfy this we will therefore assume that the mean axial velocity is strictly positive and subsonic $\left(0<\bar{v}_{x}<\bar{c}\right)$. From Eq. $(10)$ it can also be seen that the matrix $\left(\omega \bar{A}^{-1}-k_{z} \bar{A}^{-1} \bar{C}\right)$ to which we seek eigenvalues is only nontrivial when $\omega$ and $k_{z}$ are not both zero at the same time. This special case represents the circumferentially+temporally averaged mean flow, which will be treated separately in the construction of the nonreflecting interface.

If the eigenvalue problem in Eq. (10) is solved we will obtain a set of five eigenvalues, $k_{x, i}$, and corresponding eigenvectors, $\hat{q}^{i}$, which may be inserted together with the given pair of $\omega$ and $k_{z}$ in Eq. (7) to obtain all waves that are allowed to exist according to Eq. (8). The blade row interface presented in this work is based on decomposing each flow perturbation caused by a single time-azimuthal Fourier coefficient into these five waves. The direction of propagation of these waves relative to the interface, calculated based on the axial wave numbers, is then used to construct a nonreflecting exterior state on both sides of the interface. Before this can be done, however, we must define the eigenvalues/eigenvectors.

\section{Convected Waves}

The first three eigenvalues of the matrix $\left(\omega \bar{A}^{-1}-k_{z} \bar{A}^{-1} \bar{C}\right)$ are all equal and read as follows

$$
k_{x, 1}=k_{x, 2}=k_{x, 3}=\frac{\omega-k_{z} \bar{v}_{\theta}}{\bar{v}_{x}}
$$

It turns out that one can find three orthogonal eigenvectors corresponding to this eigenvalue, which in this work have been chosen as follows

$$
\hat{q}^{1}=\left[\begin{array}{c}
\bar{\rho} \\
0 \\
0 \\
0 \\
0
\end{array}\right] \quad \hat{q}^{2}=\left[\begin{array}{l}
0 \\
0 \\
\bar{c} \\
0 \\
0
\end{array}\right] \quad \hat{q}^{3}=\left[\begin{array}{c}
0 \\
-\bar{c} k_{z} \\
0 \\
\bar{c} k_{x, 1} \\
0
\end{array}\right]
$$

These eigenvectors respectively represent an entropy wave, radial vorticity wave and axial-circumferential vorticity wave. Since both the frequency and azimuthal wave numbers are real, the rate at which these waves propagate may be determined by examining the group velocity, whose axial component is given by

$$
v_{g, i}=\frac{\partial \omega}{\partial k_{x, i}}=\left(\frac{\partial k_{x, i}}{\partial \omega}\right)^{-1}=\bar{v}_{x}, \quad i=1,2,3
$$

From this we may conclude that the entropy and both vorticity waves are convected downstream with the flow, as would be expected. 


\section{Acoustic Waves}

The remaining two eigenvalues are given by

$$
\begin{aligned}
& k_{x, 4}=\frac{-\bar{v}_{x}\left(\omega-k_{z} \bar{v}_{\theta}\right)+\bar{c} \sqrt{\Lambda}}{\bar{c}^{2}-\bar{v}_{x}^{2}} \\
& k_{x, 5}=\frac{-\bar{v}_{x}\left(\omega-k_{z} \bar{v}_{\theta}\right)-\bar{c} \sqrt{\Lambda}}{\bar{c}^{2}-\bar{v}_{x}^{2}}
\end{aligned}
$$

where

$$
\Lambda=\left(\omega-k_{z} \bar{v}_{\theta}\right)^{2}-k_{z}^{2}\left(\bar{c}^{2}-\bar{v}_{x}^{2}\right)
$$

The corresponding eigenvectors to $k_{x, 4}$ and $k_{x, 5}$ have been chosen as follows

$$
\hat{q}^{4}=\left[\begin{array}{c}
\bar{\rho} \\
-\bar{c}^{2} k_{x, 4} \\
\bar{v}_{x} k_{x, 4}-\bar{v}_{x} k_{x, 1} \\
0 \\
-\bar{c}^{2} k_{z} \\
\overline{\bar{v}}_{x} k_{x, 4} \overline{\bar{\nu}}_{x} k_{x, 1} \\
\bar{\rho} \bar{c}^{2}
\end{array}\right] \quad \hat{q}^{5}=\left[\begin{array}{c}
\bar{\rho} \\
-\bar{c}^{2} k_{x, 5} \\
\overline{\bar{v}_{x} k_{x, 5}-\bar{v}_{x} k_{x, 1}} \\
0 \\
-\bar{c}^{2} k_{z} \\
\overline{\bar{v}}_{x} k_{x, 5} \overline{\bar{v}}_{x} k_{x, 1} \\
\bar{\rho} \bar{c}^{2}
\end{array}\right]
$$

These eigenvectors correspond to two acoustic waves which are either propagating (cut-on) or exponentially decaying (cut-off). The switch between these two states is determined by the factor $\Lambda$ in Eq. [15]. If it is positive, the wave numbers in Eq. (14) will be real and the waves will consequently propagate with an axial group velocity given by

$$
\begin{aligned}
& v_{g, 4}=-\frac{\bar{c}^{2}-\bar{v}_{x}^{2}}{\bar{v}_{x}-\frac{\bar{c}\left(\omega-k_{z} \bar{v}_{\theta}\right)}{\sqrt{\Lambda}}} \\
& v_{g, 5}=-\frac{\bar{c}^{2}-\bar{v}_{x}^{2}}{\bar{v}_{x}+\frac{\bar{c}\left(\omega-k_{z} \bar{v}_{\theta}\right)}{\sqrt{\Lambda}}}
\end{aligned}
$$

For the subsonic axial mean flow assumed in this work it is possible to prove that one of these group velocities will be positive and the other one negative, corresponding to one upstream and one downstream traveling wave. In cases where $\Lambda$ is negative, Eq. (14) will instead yield two complex axial wave numbers, one with positive and one with negative imaginary part. This corresponds to one wave decaying exponentially in the negative $x$ direction and one in the positive $x$ direction. In this work we assume that the direction in which a cut-off wave is decaying is the same as the direction of propagation of the wave. It must however be pointed out that the topic of causality of waves is much deeper than this [15], and the assumption made in this work is therefore not to be considered a complete answer to the question of where the waves originate.

The special case for when $\Lambda=0$ is sometimes referred to as acoustic resonance [16]. This case can be seen to yield two identical eigenvalues in Eq. (14) which in turn results in two collinear eigenvectors in Eq. (16). In order to avoid this, and thus keep the two acoustic waves unique for all situations, we have adopted the method proposed by Frey et al. [16], which will be outlined next.

\section{Extension to Account for Acoustic Resonance}

The method proposed in [16] for handling acoustic resonance is based on adding a small amount of damping to Eq. [8] according to

$$
\frac{\partial q^{\prime}}{\partial t}+\bar{A} \frac{\partial q^{\prime}}{\partial x}+\bar{C} \frac{\partial q^{\prime}}{\partial z}+\varepsilon q^{\prime}=0
$$

Here, $\varepsilon>0$ is some small parameter that sets the amount of damping. The result of adding damping to the governing equation is that the acoustic waves satisfying Eq. (18) always will be cut-off and unique, thus preventing the acoustic resonance solution from existing. To see this we proceed by inserting Eq. (7) into Eq. (18) and multiply the result by $\bar{A}^{-1}$ to obtain the following new eigenvalue problem 


$$
\left((\omega-i \varepsilon) \bar{A}^{-1}-k_{z} \bar{A}^{-1} \bar{C}-k_{x} I\right) \hat{q}(r)=0
$$

This equation can also be obtained by replacing the frequency in Eq. 10 by the following, modified frequency

$$
\tilde{\omega}=\omega-i \varepsilon
$$

Consequently, the solution to Eq. (19) may be obtained by replacing $\omega$ by $\tilde{\omega}$ in all the formulas previously provided. If this is done, it is found that the factor $\Lambda$ becomes

$$
\Lambda(\tilde{\omega})=\underbrace{\left(\omega-k_{z} \bar{v}_{\theta}\right)^{2}-k_{z}^{2}\left(\bar{c}^{2}-\bar{v}_{x}^{2}\right)}_{\Lambda(\omega)}-\varepsilon^{2}-2 \varepsilon\left(\omega-k_{z} \bar{v}_{\theta}\right) i
$$

This factor never becomes zero and thus ensures that we always obtain two unique acoustic eigenvalues when $\tilde{\omega}$ is used in Eq. [14. To prove this, assume that $\Lambda(\tilde{\omega})=0+0 i$ under some circumstance. This implies that $\omega-k_{z} \bar{v}_{\theta}=0$ for the imaginary part to be zero. In this case, the real part of $\Lambda(\tilde{\omega})$ can be seen to be strictly negative, which contradicts the original assumption. The fact that both eigenvalues always remain complex, and thus correspond to cut-off waves, have not been proven in this work but the interested reader is instead referred to [16].

In [16] it is also shown that the group velocity of a wave that would be cut-on according to the original theory coincides with the direction of damping of the wave after the frequency has been modified according to Eq. 20]. This means that we can use the sign of the imaginary part of the axial wave number to determine the direction of propagation of all the acoustic waves, which is the strategy adopted in this work. The modification of the frequency defined in Eq. (20) is further applied consistently to both the convected and acoustic waves with a damping factor equal to $\varepsilon=10^{-3} \bar{c} / P$, where $P$ the pitch of the blade row. A similar value of the damping factor has previously been used in [1, 16]. The mean axial velocity is however still used to determine the direction of propagation of the convected waves. It should also be noted that the modified frequency should not be applied in Eq. (3) since the modification only applies when the eigenvalue problem is being solved.

\section{Left Eigenvectors}

The eigenvectors derived in the previous sections may be conveniently grouped into a matrix as follows

$$
T=\left[\begin{array}{ccccc}
\bar{\rho} & 0 & 0 & \bar{\rho} & \bar{\rho} \\
0 & 0 & -\bar{c} k_{z} & \frac{-\bar{c}^{2} k_{x, 4}}{\bar{v}_{x} k_{x, 4}-\bar{v}_{x} k_{x, 1}} & \frac{-\bar{c}^{2} k_{x, 5}}{\bar{v}_{x} k_{x, 5}-\bar{v}_{x} k_{x, 1}} \\
0 & \bar{c} & 0 & 0 & 0 \\
0 & 0 & \bar{c} k_{x, 1} & \frac{-\bar{c}^{2} k_{z}}{\bar{v}_{x} k_{x, 4}-\bar{v}_{x} k_{x, 1}} & \frac{-\bar{c}^{2} k_{z}}{\bar{v}_{x} k_{x, 5}-\bar{v}_{x} k_{x, 1}} \\
0 & 0 & 0 & \bar{\rho} \bar{c}^{2} & \bar{\rho} \bar{c}^{2}
\end{array}\right]
$$

The inverse of $T$ contains the left eigenvectors to the matrix $\left(\tilde{\omega} \bar{A}^{-1}-k_{z} \bar{A}^{-1} \bar{C}\right)$. As will be shown later these vectors may be used to measure the amplitude of the waves that constitute a flow perturbation caused by a certain time-azimuthal Fourier coefficient. Before this is done, however, it should be questioned whether $T$ always remains invertible and well defined. As noted before, acoustic resonance will make the two acoustic eigenvectors collinear and thus prevent $T$ from having full rank. If it is assumed that acoustic resonance does not occur, and the modification to $\omega$ defined in Eq. (20) is not used, it is a fairly simple task to prove that $T \mathbf{x}=0$ only has trivial solutions under the assumptions stated previously. In addition to this, it can also be proven that all columns in $T$ remain well defined in the sense that they neither become a zero vector, nor include a division by zero. In this work we do however want to include the modification proposed in [16] to be able to deal with acoustic resonance points as well. As discussed previously, this modification in itself does indeed prevent acoustic resonance from causing issues. Whether the matrix $T$ still remains invertible and well defined under all other circumstances is however unknown to the authors. It is believed that this is the case, but it might happen that the introduction of $\varepsilon$ requires some additional constraints that have not been considered in this work. It should also be pointed out that the structure of the eigenvectors adopted in this work is the same as used in [14]. 
Under the assumption that $T$ is invertible the left eigenvectors may be obtained from the rows of $T^{-1}$, which read as follows

$$
T^{-1}=\left[\begin{array}{ccccc}
1 & 0 & 0 & 0 & \frac{-1}{\bar{\rho}} \bar{c}^{2} \\
0 & 0 & \frac{1}{\bar{c}} & 0 & 0 \\
0 & \frac{-k_{z}}{\overline{\bar{c}}\left(k_{x, 1}^{2}+k_{z}^{2}\right)} & 0 & \frac{k_{x, 1}}{\bar{c}\left(k_{x, 1}^{2}+k_{z}^{2}\right)} & \frac{-k_{z}}{\bar{\rho} \bar{c} \bar{v}_{x}\left(k_{x, 1}^{2}+k_{z}^{2}\right)} \\
0 & \frac{-\bar{v}_{x}\left(k_{x, 4} k_{x, 1}-k_{x, 1}^{2}\right)}{2 \bar{c}^{2}\left(k_{x, 4} k_{x, 1}+k_{2}^{2}\right)} & 0 & \frac{-\bar{v}_{x}\left(k_{x, 4} k_{z}-k_{x, 1} k_{z}\right)}{2 \bar{c}^{2}\left(k_{x, 4} k_{x, 1}+k_{z}^{2}\right)} & \frac{1}{2 \bar{\rho} \bar{c}^{2}} \\
0 & \frac{-\bar{v}_{x}\left(k_{x, 5} k_{x, 1}-k_{x, 1}^{2}\right)}{2 \bar{c}^{2}\left(k_{x, 5} k_{x, 1}+k_{z}^{2}\right)} & 0 & \frac{-\bar{v}_{x}\left(k_{x, 5} k_{z}-k_{x, 1} k_{z}\right)}{2 \bar{c}^{2}\left(k_{x, 5} k_{x, 1}+k_{z}^{2}\right)} & \frac{1}{2 \bar{\rho} \bar{c}^{2}}
\end{array}\right]
$$

In the next section we will combine the left and right eigenvectors to construct the nonreflecting interface.

\section{Construction of Nonreflecting Rotor-Stator Interface}

The construction of the nonreflecting rotor-stator interface begins by sampling the flow along bands of constant radius on both sides of the interface according to Eq. (4). The temporal Fourier coefficients used in this equation are further obtained by sampling the primitive flow variables converted to the absolute frame of reference and using the relative blade passing frequency in Eq. (2) as the dual variable. If the node distribution on both sides of the interface does not match in the radial direction, as illustrated in Fig. 1. an interpolation step is also performed. In this case, an area-weighted average of the Fourier coefficients representing the exterior solution is formed to obtain a new set of Fourier coefficients at the same radial location that the interior solution was sampled. This procedure is also illustrated in Fig. 1 for the case when the A-Side of the interface represents the interior solution. When an exterior state to the B-Side cells is calculated the picture is naturally mirrored.

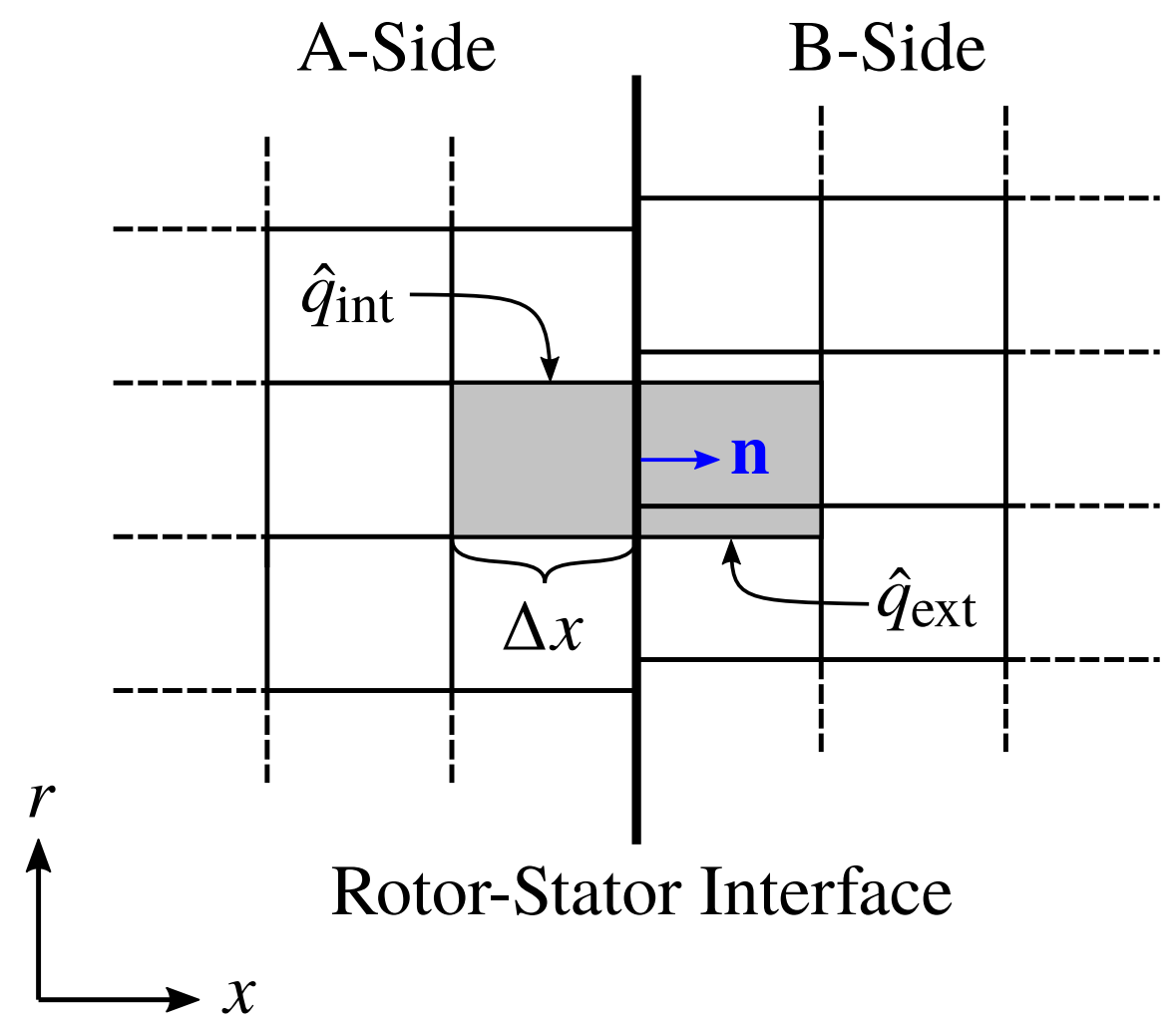

Fig. 1 Illustration of the different parameters that are used to construct ghost cell values that yield a nonreflecting exterior state for the A-Side cells. The situation is mirrored when ghost cell values for the B-Side cells are being calculated. 
The flow perturbations caused by a time-azimuthal Fourier coefficients sampled at the interior and exterior $\left(q_{\text {int }}^{\prime}\right.$, $q_{\mathrm{ext}}^{\prime}$ ) may in the absolute frame of reference be written respectively as follows

$$
\begin{aligned}
q_{\text {int }}^{\prime}\left(x_{\text {int }}, r, z, t\right) & =\hat{q}_{\text {int }}\left(x_{\text {int }}, r\right) e^{i\left(\omega t-k_{z} z\right)} \\
q_{\text {ext }}^{\prime}\left(x_{\mathrm{ext}}, r, z, t\right) & =\hat{q}_{\mathrm{ext}}\left(x_{\mathrm{ext}}, r\right) e^{i\left(\omega t-k_{z} z\right)}
\end{aligned}
$$

In this equation, $k_{z}=m_{n, k} / r$ where $m_{n, k}$ is the nodal diameter of the flow perturbation defined in Eq. (1). In the absolute frame the frequency will further read as follows

$$
\omega_{n, k}^{\mathrm{abs}}=k N_{1} \Omega_{1}+n N_{2} \Omega_{2}
$$

This frequency has been obtained by performing a Doppler shift of a flow perturbation in Eq. (3) to the absolute frame of reference. The reason for doing this is that we perform the nonreflecting analysis in the absolute frame. Alternatively, one can choose to perform the analysis in the relative frame of each individual blade row. In this case, the tangential velocity of the exterior solution must first be converted to the relative frame of the interior solution before it is sampled. In either case, the nonreflecting analysis will give the same result since the factor $\tilde{\omega}-k_{z} \bar{v}_{\theta}$ remains the same in each frame of reference. Note also that if the corresponding Fourier coefficient obtained in the interior is not available on the exterior side, we set $\hat{q}_{\mathrm{ext}}=0$.

The flow perturbations in Eq. (24) may also be expressed as a linear combination of the waves in Eq. (7) as follows

$$
\begin{aligned}
q_{\mathrm{int}}^{\prime}\left(x_{\mathrm{int}}, r, z, t\right) & =T(r) \alpha_{\mathrm{int}} e^{i\left(\omega t-k_{x} x_{\mathrm{int}}-k_{z} z\right)} \\
q_{\mathrm{ext}}^{\prime}\left(x_{\mathrm{ext}}, r, z, t\right) & =T(r) \alpha_{\mathrm{ext}} e^{i\left(\omega t-k_{x} x_{\mathrm{ext}}-k_{z} z\right)}
\end{aligned}
$$

Here, the vectors $\alpha_{\text {int }}$ and $\alpha_{\text {ext }}$ contain the amplitudes of each individual wave and the notation $T(r)$ is used to indicate that $T$ varies with $r$. The mean flow state used to calculate $T(r)$ is further defined as

$$
\bar{q}(r)=\frac{\hat{q}_{0,0, \text { int }}\left(x_{\mathrm{int}}, r\right)+\hat{q}_{0,0, \mathrm{ext}}\left(x_{\mathrm{ext}}, r\right)}{2}
$$

By equating Eqs. 24) and (26) we obtain the following relations

$$
\begin{aligned}
\hat{q}_{\text {int }}\left(x_{\mathrm{int}}, r\right) & =T(r) \alpha_{\text {int }}^{*} \\
\hat{q}_{\mathrm{ext}}\left(x_{\mathrm{ext}}, r\right) & =T(r) \alpha_{\mathrm{ext}}^{*}
\end{aligned}
$$

in which the axial dependence of the waves have been absorbed into the amplitude vectors by defining $\alpha_{\text {int }}^{*}=\alpha_{\text {int }} e^{-i k_{x} x_{\text {int }}}$

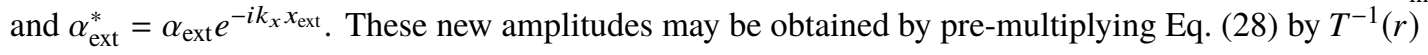

$$
\begin{aligned}
& \alpha_{\text {int }}^{*}=T^{-1}(r) \hat{q}_{\text {int }}\left(x_{\text {int }}, r\right) \\
& \alpha_{\text {ext }}^{*}=T^{-1}(r) \hat{q}_{\text {ext }}\left(x_{\text {ext }}, r\right)
\end{aligned}
$$

The purpose of the nonreflecting interface is now to specify a new exterior state which contains the outgoing waves taken from the interior plus the incoming waves taken from the exterior. This will drive the interior solution towards a state that only contains inwards propagating waves that exist at the exterior. This yields a perfectly nonreflecting interface in cases when the flow at the interface obeys Eq. (5). In other cases, the converged solution at the interface will attain a state which causes some reflections due to the fact that the interior solution contains waves that are not described by Eq. (5), thus making the decomposition into incoming and outgoing waves incomplete. A detailed description of how the absorbing exterior state is constructed is provided below. Note that we have split the description into two parts, one regarding the non-zero Fourier coefficients (for which $\omega$ and $k_{z}$ are both not zero at the same time) and one for the mean flow.

\section{Non-zero Fourier Coefficients}

The algorithm for constructing a nonreflecting exterior state for the non-zero time-azimuthal Fourier coefficients is presented as pseudo-code in Algorithm 1 This algorithm is executed once for each Fourier coefficient (corresponding to a certain pair of $\omega$ and $k_{z}$ ) at each radial location on both sides of the interface. Before it can be executed the Fourier coefficients on both sides of the interface must be calculated for all radial locations and, in cases the grid nodes do not match in the radial direction, also be interpolated so that an exterior solution at the same radial location as the 
interior solution is available. If no Fourier coefficient is available from the other side of the interface, $\hat{q}_{\text {ext }}$ is set to the zero vector. In addition to this, the x-component of the face normal for the interior face $\left(n=\mathbf{n} \cdot \mathbf{e}_{x} /\left\|\mathbf{n} \cdot \mathbf{e}_{x}\right\|\right)$, a representative length of the cell closest to the interface $(\Delta x)$ and a number that represents the minimum number of points per wavelength $(N)$ required for convected waves to be included in the analysis, must be supplied to the algorithm. The parameters supplied to Algorithm 1] are also illustrated in Fig. 1] In G3D::Flow, boundary conditions are specified using two ghost cell layers. Consequently, the output of the algorithm is a new time-azimuthal Fourier coefficient that defines a nonreflecting exterior state for the first $\left(\hat{q}_{\mathrm{abs}}\left(x_{1}\right)\right)$ and second $\left(\hat{q}_{\mathrm{abs}}\left(x_{2}\right)\right)$ ghost cell layer. These coefficients will later be converted to primitive variables using Eq. (3) in order to compute the flux which will be added to the interior cells at that radial location. Some important steps of Algorithm 1 will now be explained in more detail.

In step 3 of Algorithm 1 the convected waves are only included in the analysis if their axial wave number is sufficiently small. This check is included since $k_{x, 1}$ is proportional to the reciprocal of $\bar{v}_{x}$ and thus can grow very large in regions with low velocities, such as close to walls. This will in turn yield large oscillations in the modal amplitudes of the waves when they are phase-shifted to the locations of the ghost cells in step 7 or 12, which in turn could result in numerical instabilities. The limit on the axial wave number depends on the factor $N$, which should represent the minimum number of points per wavelength necessary for the numerical scheme employed to not cause excessive numerical dissipation. As

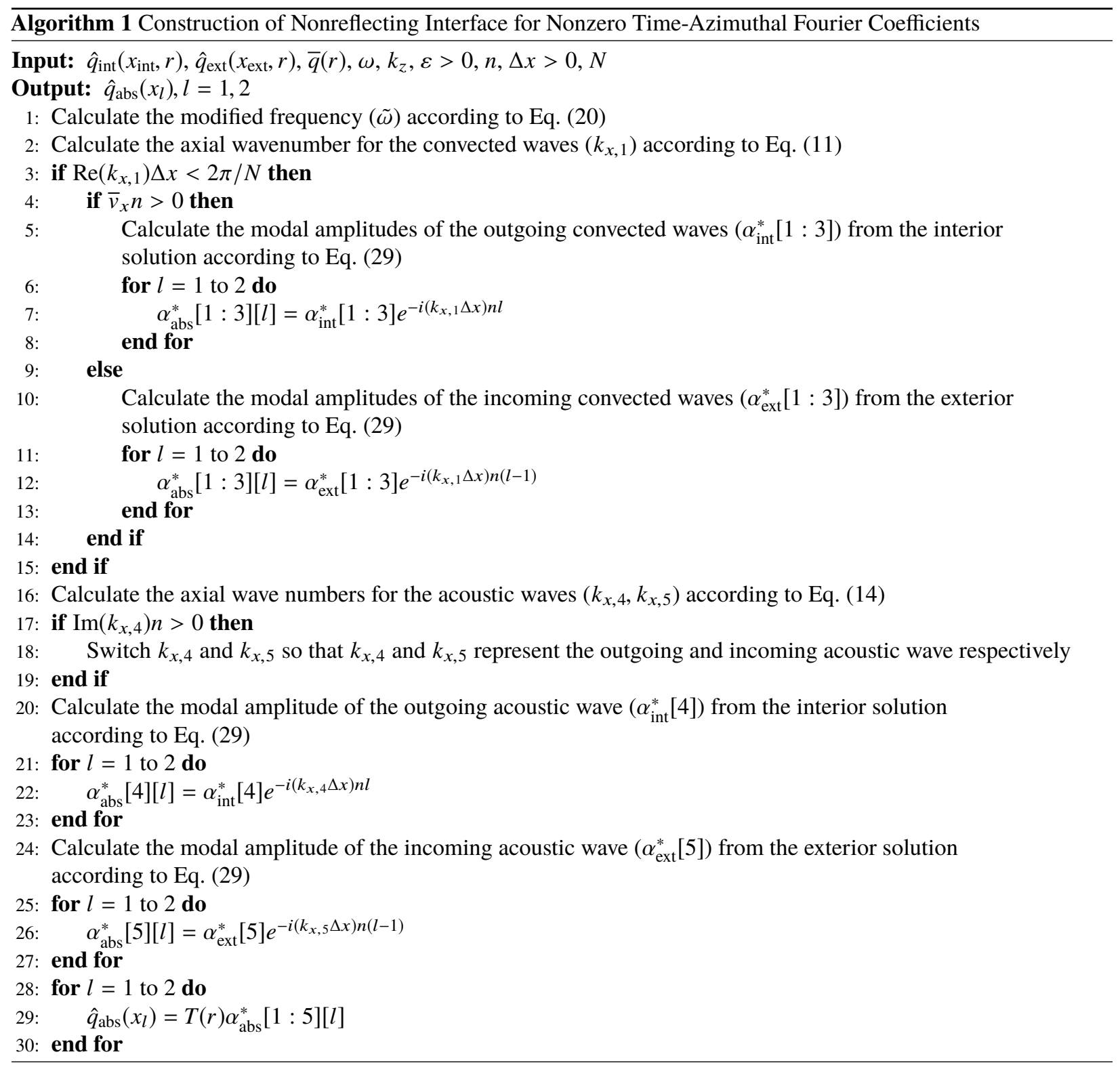


such, only convected waves which are are not resolved properly by the computational mesh will be excluded from the nonreflecting analysis, and step 3 of Algorithm 1 is therefore not expected to yield any significant errors. Note that the phase-shift employed here only is necessary for cell-centered solvers, since for a node centered scheme the information is already available at the boundary [17].

In step 17 of Algorithm 1 a check is made to determine if the first acoustic wave is incoming. If this is true, a sorting step is performed so that $k_{x, 4}$ and $k_{x, 5}$ represent the outgoing and incoming acoustic wave for the remainder of the algorithm. Due to the definition of the eigenvectors in Eqs. (22) and (23), this will also cause the fourth and fifth column/row in $T(r)$ and $T^{-1}(r)$ to represent the outgoing and incoming wave as well.

The implementation of Algorithm 1 in G3D::Flow was found to cause numerical instabilities for some cases. Typically, it was found that when either a vorticity wave or an acoustic wave impinged on the interface a slowly growing instability was formed which eventually caused the solution to diverge, even in cases when the solution appeared to converge well at the onset of the iteration process. Further investigation revealed that the instability caused a flow field which resembled a cut-off wave. This lead the authors to implement a fix which excludes waves that are strongly damped (large imaginary part of acoustic wave numbers) from the analysis. This was found to help convergence considerably, although residuals approaching machine-zero were never obtained in cases containing vorticity or acoustic waves. In addition, simulations employing less dissipative schemes that the third order upwind scheme used in this work were found to not converge even when the fix was employed. It is therefore believed that the currently proposed implementation of the interface in which the incoming waves are directly prescribed is ill-posed when a pseudo-time evolution process is used to converge the residual, as is done in G3D::Flow. This hypothesis is consistent with what has been suggested previously by other authors [1,7].

\section{Mean Flow}

As mentioned earlier the mean flow is not treated by the nonreflecting analysis presented in the previous sections. Instead, 1D characteristic analysis is used to decompose the zeroth time-azimuthal Fourier coefficients obtained on both sides of the interface into incoming and outgoing characteristic variables. After this, the outgoing characteristic variables from the interior, extrapolated to the location of the ghost cells, and the incoming characteristic variables from the exterior are combined to form a new set of characteristic variables. This new set can finally be transformed back to obtain a new time-azimuthal Fourier coefficient for the zeroth mode which defines the nonreflecting mean flow state at the exterior. It should be emphasized that this approach for handling the mean flow not is conservative.

\section{Results}

\section{A. Propagation of Waves in a Two Dimensional Cascade}

As stated previously the purpose of the blade row interface presented in this work is to ensure that waves impinging on the interface do not reflect and, in cases when the waves are resolved on the other side of the interface, also make sure that they continue to propagate in the adjacent blade row. To test this, a two dimensional cascade consisting of three sub-domains, among which the middle one is translating in the positive y-direction, was constructed as shown in Fig. 2. Nonreflecting blade row interfaces were placed at the connections between the sub-domains. A downstream traveling wave is then excited at the upstream interface and allowed to propagate through the second sub-domain until it reaches the downstream interface. At this interface, the modal amplitudes of all incoming waves are explicitly set to zero on both sides. This way, the interface will act as a nonreflecting boundary condition and prevent the wave from being transmitted to the third sub-domain. After verifying that this works for all convected waves and a cut-on acoustic wave traveling downstream, we mirror the situation and perform the same analysis for a cut-on acoustic wave traveling upstream. Finally, we also rerun the simulation for the cut-on acoustic wave traveling downstream without setting the modal amplitudes to zero at the downstream interface in order to ensure that waves are properly transmitted across it. In this case we also employ a buffer layer as indicated by the shaded region in Fig. 2 to damp out the wave before it reaches the outlet and cause any reflections. Details on the implementation of the buffer layer is presented in [18].

In all simulations the flow is modeled using the nonlinear Euler equations coupled with an ideal gas equation of state and the thermodynamic properties are taken to be those of air. Inviscid fluxes are computed by upwinding 1D characteristic variables to the cell faces using a third-order accurate scheme [19]. Further details on the computational setup are provided in Table 2 The frequency and azimuthal wave number provided in this table have been calculated based on the two dimensional forms of Eqs. (25) and (1), which read as follows 


$$
\begin{array}{ll}
\omega_{n, k}^{\mathrm{abs}}=k \frac{2 \pi}{P_{1}} v_{d, 1} & +n \frac{2 \pi}{P_{2}} v_{d, 2} \\
k_{z}^{n, k}=k \frac{2 \pi}{P_{1}} & +n \frac{2 \pi}{P_{2}}
\end{array}
$$

Here, $P$ and $v_{d}$ represent the pitch and the translational velocity of the blade row. All simulations performed in this work have been run with $n=2$ and $k=-1$ in Eq. (30) which means that the translational velocity becomes the sole variable determining the frequency. Also note that the frequencies reported in Table 2 are valid for the wave in the absolute frame of reference. The corresponding frequencies in the relative frame of reference of the second sub-domain may be calculated as $\omega_{k, 2}=-k \frac{2 \pi}{P_{1}} v_{d, 2}$. It should also be noted that the azimuthal wave number used does not yield waves which are periodic in the y-direction with the same period as the pitch of the blade row. To account for this, phase shifted periodic boundary conditions are employed at the pitchwise boundaries [11, 20].

The pressure perturbations caused by a cut-on acoustic wave propagating downstream is illustrated in Fig. 3 for the case when the wave is absorbed at the downstream interface (3a) and when the wave is transmitted across the interface (3b). The two pictures appear identical and show no signs of unphysical reflections or distortion of the wave close to the downstream interface. In order to get more details on the performance of the interface the flow fields obtained for the waves defined in Table 2 were also extracted along an axial line going through the second sub-domain. These results have then been compared against the corresponding analytical solution to verify that the waves are properly imposed, propagated, and absorbed.

Results obtained for the entropy wave are presented in Fig. 4 From this figure it can be seen that the numerical and analytical solutions agree very well and that no signs of reflections can be seen at the downstream interface located at $x=9 \mathrm{~m}$. Furthermore, no perturbations in the other flow field variables were observed in the simulation, which shows that the implementation indeed excites and absorbs the entropy wave correctly.

Next, the velocity perturbations caused by the axial-circumferential vorticity wave is presented in Fig. 5. Once again, the agreement between the numerical and analytical solution is very good for both the axial and tangential velocity component. This shows that the code handles vorticity waves correctly as well. At this point it must however be pointed out that convergence only could be reached if strongly damped acoustic waves were excluded in the analysis. The fact that this problem occurs when a vorticity wave is simulated may be connected to the fact that a velocity perturbation can yield a non-zero amplitude for an acoustic wave in Eq. 29] if the velocity perturbation does not stem from a pure vorticity wave. At the onset of the iteration process, such a velocity perturbation may very well exist in the flow, which could explain why the seemingly unstable acoustic waves were excited at the interface.

The perturbations in axial velocity and pressure induced by the downstream traveling acoustic wave are presented in Fig. 6. The agreement between the numerical and analytical results is once again very good, with only small deviations appearing close to the downstream interface. These deviations are partly due to the dissipation and dispersion introduced by the numerical scheme, and probably also partly be due to the suspected ill-posedness of the chosen implementation strategy.

Larger numerical dissipation and dispersion errors are seen in Fig.77for the cut-on acoustic wave traveling upstream. This result is expected since the upstream traveling wave has a significantly shorter wave length compared to the downstream traveling one in Fig. 6. Despite this, the numerical results are in good agreement with respect to the amplitude and phase of both the axial velocity and pressure perturbations.

Finally, the results obtained for the downstream traveling acoustic wave when the downstream interface allows the wave to propagate through is presented in Fig. 8. Most importantly, it can be seen that the wave propagates without getting distorted or reflected past the position of the interface at $x=9 \mathrm{~m}$. This shows that the mode matching indeed works correctly. 


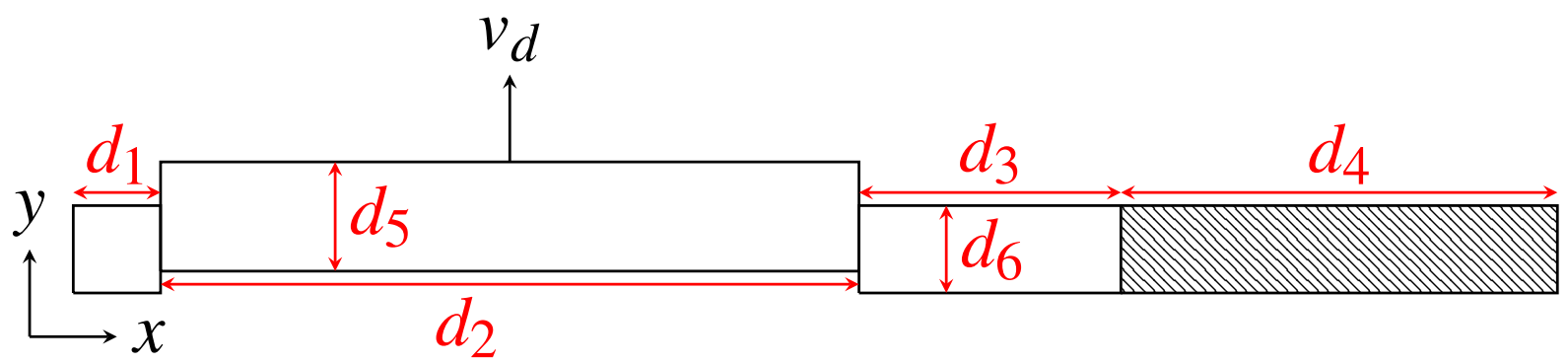

Fig. 2 Schematic view of the computational domain used for verification. The sizes shown are listed in Table 1

Table 1 Size of computational domain presented in Fig. 2 .

\begin{tabular}{cccccc}
$d_{1}$ & $d_{2}$ & $d_{3}$ & $d_{4}$ & $d_{5}$ & $d_{6}$ \\
$1 \mathrm{~m}$ & $8 \mathrm{~m}$ & $3 \mathrm{~m}$ & $5 \mathrm{~m}$ & $1.25 \mathrm{~m}$ & $1 \mathrm{~m}$ \\
\hline
\end{tabular}

Table 2 Numerical setup used for verification.

(a) Inlet

\begin{tabular}{lll}
\hline Variable & Value & Unit \\
$\bar{\rho}$ & 1.4 & $\mathrm{~kg} \mathrm{~m}^{-3}$ \\
$\bar{v}_{x}$ & 100 & $\mathrm{~m} \mathrm{~s}^{-1}$ \\
$\bar{v}_{y}$ & -20 & $\mathrm{~m} \mathrm{~s}^{-1}$ \\
$\bar{p}$ & 40000 & $\mathrm{~kg} \mathrm{~m}^{-1} \mathrm{~s}^{-2}$ \\
\hline
\end{tabular}

(c) Convected Waves

\begin{tabular}{lll}
\hline Variable & Value & Unit \\
$v_{d}$ & 50 & $\mathrm{~m} \mathrm{~s}^{-1}$ \\
$\omega$ & 502.65 & $\mathrm{rad} \mathrm{s}^{-1}$ \\
$k_{z}$ & 3.7699 & $\mathrm{rad} \mathrm{m}^{-1}$ \\
$N_{h}$ & 3 & \\
$N_{a}$ & 4 & \\
\hline
\end{tabular}

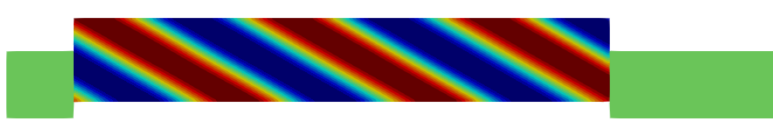

(a) Bladerow interface disabled. (b) Outlet

\begin{tabular}{lll}
\hline Variable & Value & Unit \\
$\bar{p}$ & 40000 & $\mathrm{~kg} \mathrm{~m}^{-1} \mathrm{~s}^{-2}$ \\
\hline
\end{tabular}

(d) Acoustic Waves

\begin{tabular}{lll}
\hline Variable & Value & Unit \\
$v_{d}$ & 100 & $\mathrm{~m} \mathrm{~s}^{-1}$ \\
$\omega$ & 1005.31 & $\mathrm{rad} \mathrm{s}^{-1}$ \\
$k_{z}$ & 3.7699 & $\mathrm{rad} \mathrm{m}^{-1}$ \\
$N_{h}$ & 3 & \\
$N_{a}$ & 4 & \\
\hline
\end{tabular}

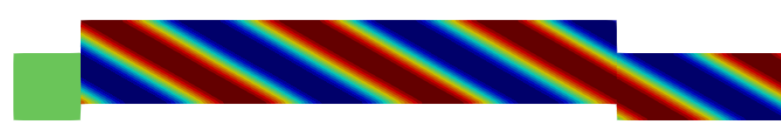

(b) Bladerow interface enabled.

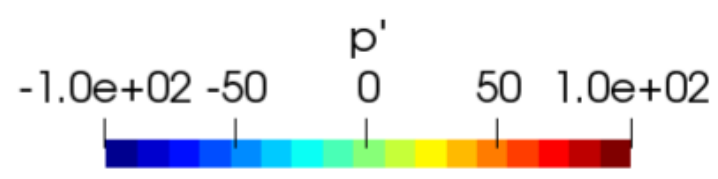

Fig. 3 Pressure fluctuations $\left(p^{\prime}, \mathrm{Pa}\right)$ for cut-on acoustic wave propagating downstream. 


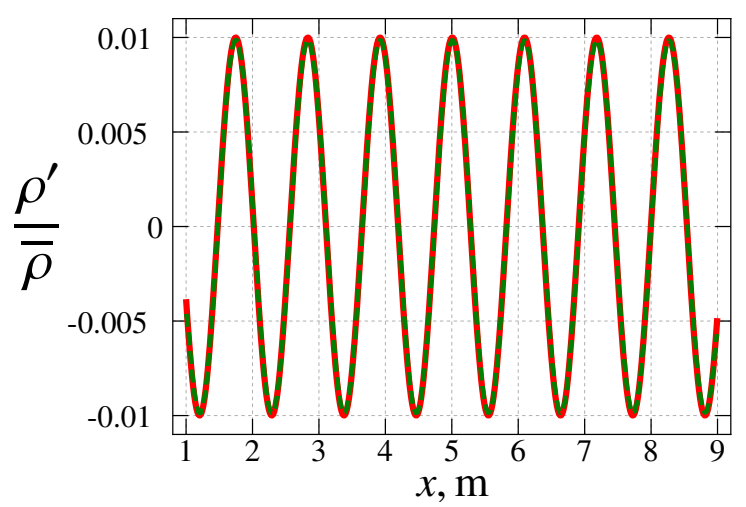

Fig. 4 Normalized density perturbation for the entropy wave (Analytical solution: - - Numerical solution: $-=-$ ).
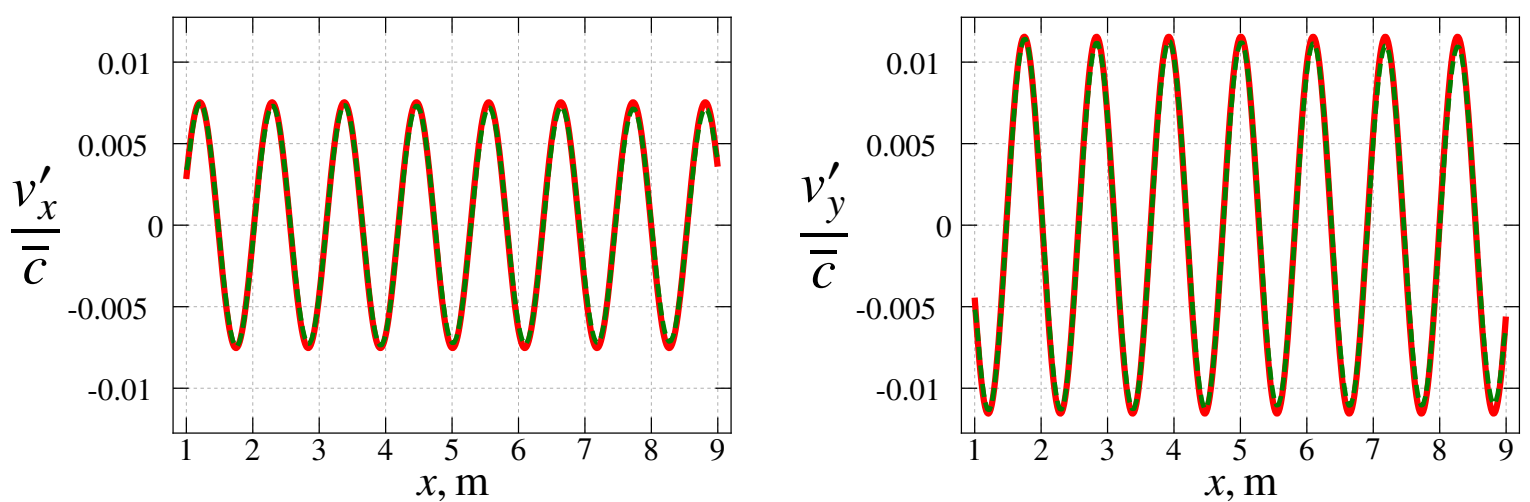

(a) Axial component of velocity perturbation normalized (b) Tangential component of velocity perturbation normalby speed of sound. ized by speed of sound.

Fig.5 Normalized velocity perturbations for the vorticity wave (Analytical solution: - Numerical solution: $----)$.
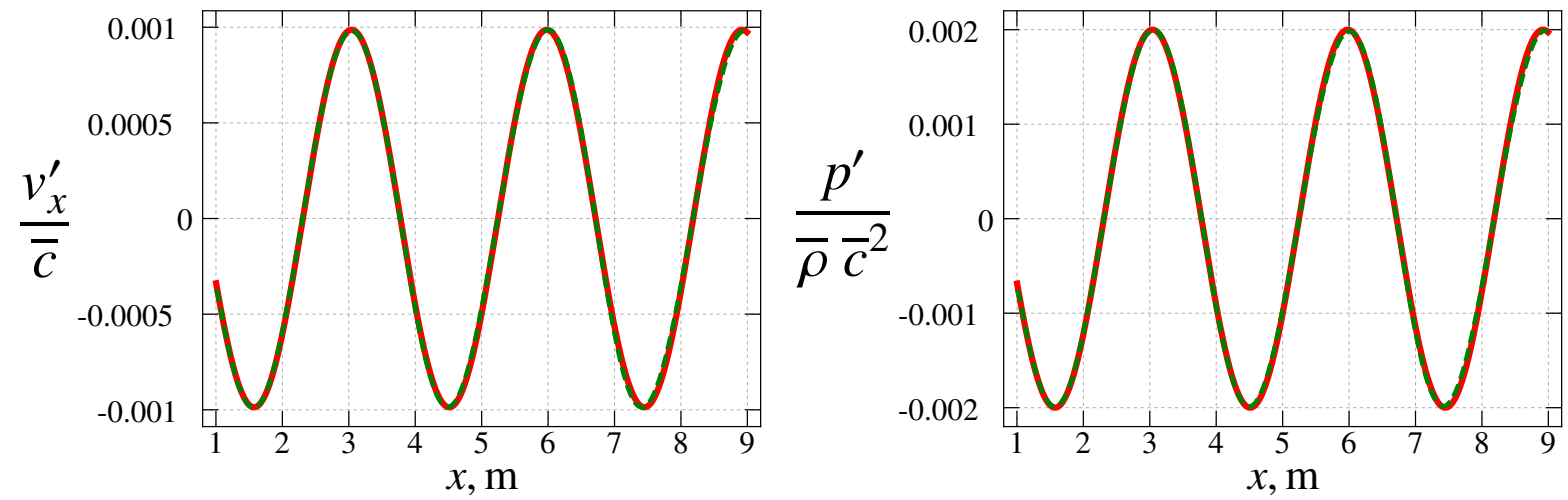

(a) Axial component of velocity perturbation normalized (b) Pressure perturbation normalized by density and speed by speed of sound. of sound.

Fig. 6 Normalized velocity and pressure perturbations for the downstream traveling acoustic wave (Analytical solution: - , Numerical solution: - - - ). 

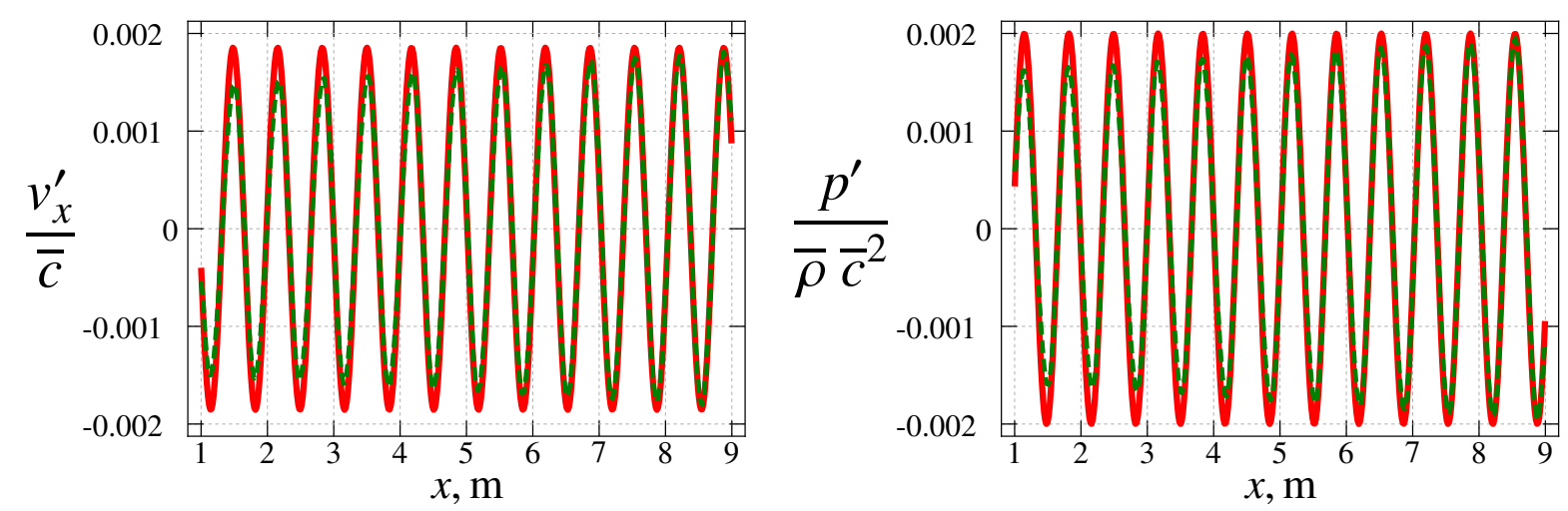

(a) Axial component of velocity perturbation normalized (b) Pressure perturbation normalized by density and speed by speed of sound. of sound.

Fig. 7 Normalized velocity and pressure perturbations for the upstream traveling acoustic wave (Analytical solution: - Numerical solution: - - - ).
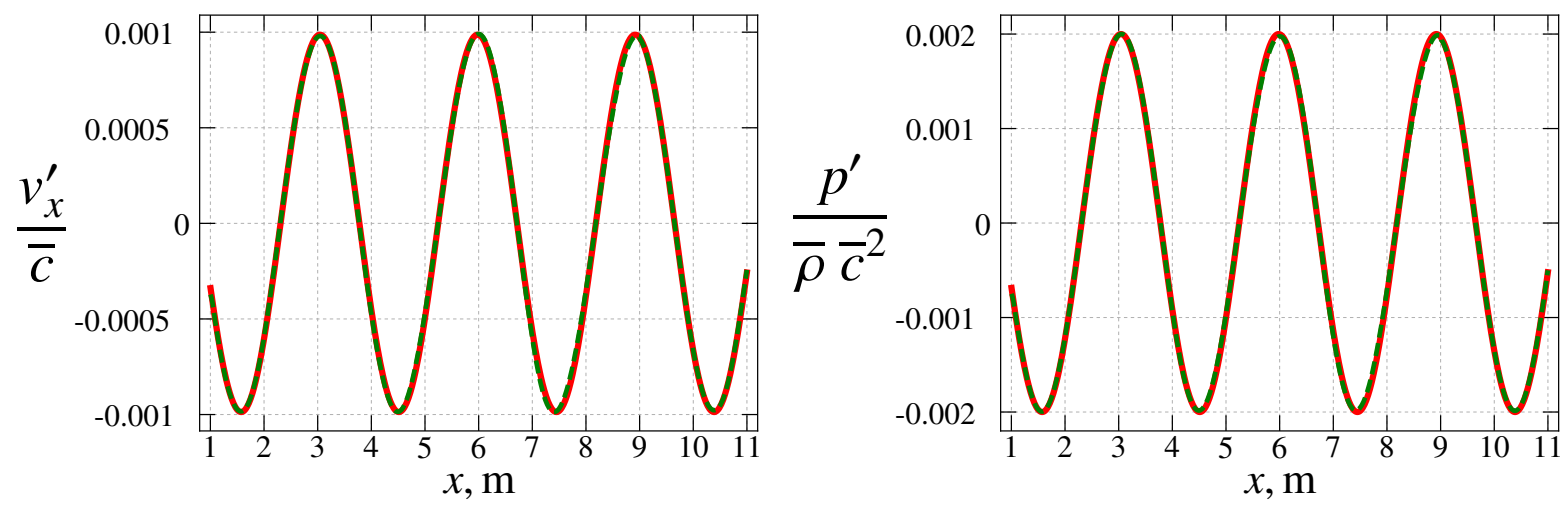

(a) Axial component of velocity perturbation normalized (b) Pressure perturbation normalized by density and speed by speed of sound. of sound.

Fig. 8 Normalized velocity and pressure perturbations for the downstream traveling acoustic wave that crosses the rotor-stator interface located at $x=9 \mathrm{~m}$ (Analytical solution: - - Numerical solution: - - - - ). 


\section{Conclusion}

In this work a nonreflecting rotor-stator interface for steady and unsteady simulations in axial turbomachines has been presented and verified for a two dimensional flow in a straight cascade. The nonreflecting interface is constructed by projecting the flow close to the interface onto incoming and outgoing waves satisfying the linearized Euler equations, as was originally propsed by Giles [6, 7] for two dimensional flows. In this work we also adopt the extension of Giles method to three dimensional flows proposed by Saxer and Giles [9], often referred to as the Quasi-Three-Dimensional approach. In addition to this, we have adopted the regularization proposed by Frey et al. [16] which ensures that waves corresponding to acoustic resonance are not allowed to exist. Our final implementation is to some extent similar to the work reported by Robens et al. [17] in which phase shifting of the waves from the interior cells is employed to obtain a definition of the exterior solution. The eigenvectors, which scale the perturbations of the primitive flow variables induced by the corresponding wave, have in this work been chosen to be the same as those reported by Kersken et al. [14].

Two dimensional wave propagation along a straight cascade, inside which two nonreflecting interfaces were placed, was chosen to verify the implementation. The results obtained from the verification revealed that the interface does not yield any visible reflections if two dimensional waves impinge on it, and that waves that are resolved on both sides of the interface can pass across it without becoming distorted. Simulations including vorticity and acoustic waves also showed a slowly growing instability which eventually caused the simulations to diverge. This problem was partially solved by excluding acoustic waves that were strongly damped from the nonreflecting analysis, as has previously been done by Olausson [5]. It is believed that the cause of the instability is that incoming waves are directly set equal to the exterior state or zero in order to enforce a nonreflecting state at the interface. This may in turn yield an ill-posed problem when a pseudo time iteration process is used to converge the residual [7], as is done in this work. The solution proposed in [7] to deal with this is to reformulate the nonreflecting condition in terms of a condition on the 1D characteristic variables. This approach has been used successfully by other authors employing a pseudo time iteration solver [1, 14, 21] and will therefore likely be investigated further in future work. In addition to this, the interface will be extended to become conservative by employing a different treatment of the mean flow similar to what has been done previously by e.g. Saxer and Giles [9] and Frey et al. [22].

Although the verification case investigated is this work was restricted to unsteady wave propagation in two dimensions, the theory covered in this work can be applied to both steady and unsteady simulations in three-dimensional axial turbomachines without modification. The theory is also directly applicable for formulating nonreflecting inlet and outlet boundary conditions. In these cases, however, the mean flow should once again be handled differently dependent on the type of mean flow boundary condition that is enforced at the boundary.

\section{Acknowledgments}

This work is financially supported by the E.U. under the ULTIMATEFProject funded by the European Commission within the Horizon 2020 Programme (2014-2020) under the Grant Agreement No 633436.

\section{References}

[1] Frey, C., Ashcroft, G., and Kersken, H.-P., "Simulations of Unsteady Blade Row Interactions Using Linear and Non-Linear Frequency Domain Methods,” ASME Turbo Expo 2015: Turbine Technical Conference and Exposition, GT2015-43453, 2015.

[2] Hall, K. C., Jeffrey, P. T., and Clark, W. S., "Computation of Unsteady Nonlinear Flows in Cascades Using a Harmonic Balance Technique,” AIAA Journal, Vol. 40, No. 5, 2002, pp. 879-886.

[3] McMullen, M. S., and Jameson, A., "The Computational Efficiency of Non-Linear Frequency Domain Methods," Journal of Computational Physics, Vol. 212, No. 2, 2006, pp. 637-661.

[4] Ekici, K., and Hall, K. C., "Nonlinear Analysis of Unsteady Flows in Multistage Turbomachines Using Harmonic Balance," AIAA Journal, Vol. 45, No. 5, 2007, pp. 1047-1057.

[5] Olausson, M., "Turbomachinery Aeroacoustic Calculations using Nonlinear Methods," Ph.D. thesis, Division of Fluid Dynamics, Department of Applied Mechanics, Chalmers University of Technology, Gothenburg, Sweden, 2011.

[6] Giles, M. B., "Nonreflecting Boundary Conditions for Euler Equation Calculations," AIAA Journal, Vol. 28, No. 12, 1990, pp. 2050-2058.

\footnotetext{
*Ultra Low emission Technology Innovations for Mid-century Aircraft Turbine Engines
} 
[7] Giles, M. B., "Non-Reflecting Boundary Conditions for the Euler Equations," CFDL-TR-88-1, 1988.

[8] Engquist, B., and Majda, A., "Absorbing Boundary Conditions for the Numerical Simulation of Waves," Mathematics of Computation, Vol. 31, No. 139, 1977, pp. 629-651.

[9] Saxer, A. P., and Giles, M. B., "Quasi-Three-Dimensional Nonreflecting Boundary Conditions for Euler Equations Calculations," Journal of Propulsion and Power, Vol. 9, No. 2, 1993, pp. 263-271.

[10] Tyler, J. M., and Sofrin, T. G., “Axial Flow Compressor Noise Studies,” SAE Technical Paper, 620532, 1962.

[11] Gerolymos, G. A., Michon, G. J., and Neubauer, J., "Analysis and Application of Chorochronic Periodicity in Turbomachinery Rotor/Stator Interaction Computations," Journal of Propulsion and Power, Vol. 18, No. 6, 2002, pp. 1139-1152.

[12] Lindblad, D., Montero Villar, G., Andersson, N., Capitao Patrao, A., Courty-Audren, S.-k., and Napias, G., "Aeroacoustic Analysis of a Counter Rotating Open Rotor based on the Harmonic Balance Method," 2018 AIAA Aerospace Sciences Meeting, AIAA Paper 2018-1004, 2018.

[13] Ekici, K., and Hall, K. C., "Nonlinear Frequency-Domain Analysis of Unsteady Flows in Turbomachinery with Multiple Excitation Frequencies,” AIAA Journal, Vol. 46, No. 8, 2008, pp. 1912-1920.

[14] Kersken, H.-P., Ashcroft, G., Frey, C., Wolfrum, N., and Korte, D., "Nonreflecting Boundary Conditions for Aeroelastic Analysis in Time and Frequency Domain 3D RANS Solvers," ASME Turbo Expo 2014: Turbine Technical Conference and Exposition, GT2014-25499, 2014.

[15] Vilenski, G. G., and Rienstra, S. W., "On hydrodynamic and acoustic modes in a ducted shear flow with wall lining," Journal of Fluid Mechanics, Vol. 583, 2007, p. 45-70.

[16] Frey, C., and Kersken, H.-P., "On the Regularisation of Non-Reflecting Boundary Conditions near Acoustic Resonance," VII European Congress on Computational Methods in Applied Sciences and Engineering, 2016.

[17] Robens, S., Jeschke, P., Frey, C., Kügeler, E., Bosco, A., and Breuer, T., "Adaptation of Giles Non-Local Non-Reflecting Boundary Conditions for a Cell-centered Solver for Turbomachinery Applications," ASME Turbo Expo 2013: Turbine Technical Conference and Exposition, GT2013-94957, 2013.

[18] Lindblad, D., and Andersson, N., "Validating the Harmonic Balance Method for Turbomachinery Tonal Noise Predictions," 55th AIAA Aerospace Sciences Meeting, AIAA Paper 2017-1171, 2017.

[19] Andersson, N., "A Study of Subsonic Turbulent Jets and Their Radiated Sound Using Large-Eddy Simulation," Ph.D. thesis, Division of Fluid Dynamics, Department of Applied Mechanics, Chalmers University of Technology, Gothenburg, Sweden, 2005.

[20] Sicot, F., Dufour, G., and Gourdain, N., “A Time-Domain Harmonic Balance Method for Rotor/Stator Interactions,” Journal of Turbomachinery, Vol. 134, No. 1, 2012, pp. 011001-1-011001-13.

[21] Schlüß, D., Frey, C., and Ashcroft, G., "Consistent Non-Reflecting Boundary Conditions for both Steady and Unsteady Flow Simulations in Turbomachinery Applications," VII European Congress on Computational Methods in Applied Sciences and Engineering, 2016.

[22] Frey, C., Kersken, H.-P., Ashcroft, G., and Voigt, C., "A Harmonic Balance Technique for Multistage Turbomachinery Applications," ASME Turbo Expo 2014: Turbine Technical Conference and Exposition, GT2014-25230, 2014. 